\title{
Nephropathy and growth hormone deficiency in a patient with mitochondrial tRNA ${ }^{\mathrm{Leu}(\mathrm{UUR})}$ mutation
}

Tohru Yorifuji, Masahiko Kawai, Toru Momoi, Hiroshi Sasaki, Kenshi Furusho, Junko Muroi, Ken Shimizu, Yasuo Takahashi, Masahiko Matsumura, Mitsuhiko Nambu, Takehiko Okuno

\begin{abstract}
A mitochondrial A 3243 G mutation in the tRNA ${ }^{\text {Lea(UUR) }}$ gene was first described as a common cause of MELAS syndrome (mitochondrial myopathy, encephalopathy, lactic acidosis, and stroke-like syndrome). This same mutation is also the cause of a totally different disorder, a subtype of diabetes mellitus which is inherited maternally and often associated with sensorineural hearing loss. In this paper, we report on a Japanese boy with A 3243 G who developed a previously undescribed combination of symptoms, nephropathy and growth hormone deficiency. The patient first presented with short stature and moderate mental retardation. Growth hormone (GH) provocation tests showed deficient growth hormone secretion. During the course of follow up, he presented with progressive nephropathy followed by the development of diabetes mellitus. The results of laboratory tests and renal biopsy were against incidental association of known types of nephropathy. On PCR-RFLP analysis, the percentage of mutated mtDNA was higher in the renal biopsy specimen than 12 peripheral blood leucocytes. Our case suggests that mitochondrial diseases should be taken into account when there is nephropathy of unknown cause. In addition, the presence of growth hormone deficiency may account for part of the mechanism leading to short stature commonly seen in these patients.

( 7 Med Genet 1996;33:621-622)
\end{abstract}

Key words: nephropathy; growth hormone deficiency; mitochondrial mutation.

The patient came to us because of short stature (mean $-2.9 \mathrm{SD}$ ) and moderate mental re-

Provocative tests for anterior pituitary hormones

\begin{tabular}{lllc}
\hline Stimuli & Hormone & Basal value & Peak value \\
\hline Insulin & GH (ng/ml) & $<1.5$ & $5 \cdot 2$ \\
& Cortisol (mg/dl) & $20 \cdot 2$ & $20 \cdot 3$ \\
Arginine & GH (ng/ml) & $<1.5$ & $5 \cdot 1$ \\
Levodopa & GH (ng/ml) & $<1.5$ & $5 \cdot 4$ \\
LHRH & LH (IU/l) & $2 \cdot 3$ & $4 \cdot 7$ \\
& FSH (IU/l) & $<1.95$ & $2 \cdot 4$ \\
TRH & TSH (mU/ml) & $0 \cdot 78$ & $3 \cdot 4$ \\
\hline
\end{tabular}

* Peak $\mathrm{GH}$ value below $10 \mathrm{ng} / \mathrm{ml}$ is usually used as evidence of deficient secretion. tardation aged 11 years. His father and older sister were healthy. His mother had had sensorineural hearing loss from childhood, and developed diabetes mellitus at the age of 29 years. $\mathrm{He}$ had some dysmorphic features including microcephaly, hypertrichosis, right simian crease, clinodactyly, and long cilia. At the age of 12 years 6 months, $\mathrm{GH}$ therapy was initiated since he had delayed bone maturation and diminished response on $\mathrm{GH}$ provocation tests (table 1). He caught up in growth after the initiation of $\mathrm{GH}$ therapy. At the age of 13

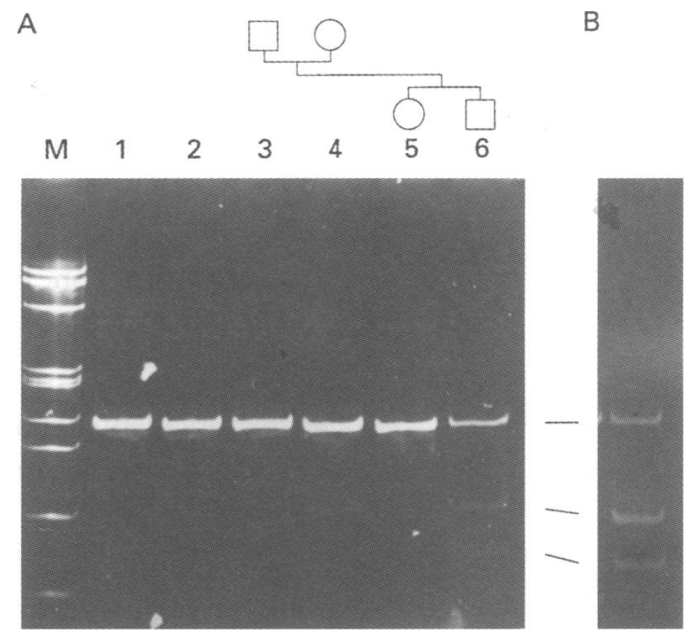

Mitochondrial mutation in the patient. Genomic DNA $(50 \mathrm{ng})$ from peripheral blood leucocytes or from renal biopsy specimen was used to amplify a $219 \mathrm{bp}$ segment within the $t R N A^{\text {Leu(UUR) }}$ gene of the mitochondrial DNA. In the presence of mitochondrial $A$ to $G$ mutation at position 3243, digestion of the $P C R$ product with restriction enzyme ApaI yields two additional fragments of $94 \mathrm{bp}$ and $125 \mathrm{bp}$. Amplifications were performed in $25 \mu \mathrm{l}$ reaction mixtures containing 10 mmol/l Tris- $\mathrm{HCl}, \mathrm{pH} 8 \cdot 3$, $50 \mathrm{mmol} / \mathrm{K} \mathrm{KCl}, 1.5 \mathrm{mmol} / \mathrm{l} \mathrm{MgCl}_{2}, 200 \mathrm{mmol} / \mathrm{l}$ each dNTPs, 25 pmol of each primer, and 1 unit of Taq DNA polymerase (Toyobo, fapan). The primer sequences were as follows: forward 5'-TTCACAAAGCGCCTTCCCCC3', reverse 5'-AGCATTAGGAATGCCATTGC-3'. The cycling parameters were as follows: initial denaturation at $94^{\circ} \mathrm{C}$ for three minutes, denaturation at $94^{\circ} \mathrm{C}$ for one minute, annealing and extension at $60^{\circ} \mathrm{C}$ for one minute for 30 cycles. Five microlitres of each PCR product were digested with ApaI and analysed by electrophoresing on a $10 \%$ polyacrylamide gel. (A) Peripheral blood leucocytes. M: $\varphi$ X174DNA digested with HaeIII. Lanes 1 and 2: normal control, 3: father, 4: mother, 5: older sister, 6: proband. (B) Renal biopsy specimen of the proband. Percentage of mutated mitochondria was estimated by scanning the gel with an image scanner (EPSON, GT$9000 A R T)$ and analysing the band intensity with the Image 1.44 program (NIH Research Service Branch).

The percentage could be underestimated because of the heteroduplex formation during PCR. 
years 1 month, proteinuria was first noted and gradually worsened to pre-renal failure status. Serum complements, immunoglobulins, and antibodies against $\beta$-streptococcus were normal. Renal biopsy showed both glomerular and tubulointerstitial changes. In the glomeruli, there were mild mesangioproliferative changes with focal sclerosis. In the tubulointerstitial region, there was mild fibrosis, infiltration of lymphocytes, and thickening of the basement membrane. Immunofluorescent study showed only mild segmental deposition of $\mathrm{C} 3 \mathrm{~d}$ in the capillary region. At the age of 14 years 3 months, glucosuria was first noted. Oral glucose tolerance test showed a diabetic pattern with diminished insulin response. GH therapy was stopped because of diabetes and since then his growth rate has decreased. Mitochondrial diabetes mellitus was suspected at this time. Audiogram showed mild sensorineural hearing disturbance. Plasma lactate and pyruvate were not raised. Electrocardiography showed type A WPW syndrome. PCR-RFLP analysis of peripheral blood leucocytes showed the presence of mitochondrial A $3243 \mathrm{G}$ in both the patient and his mother (figure). Estimated percentages of mutated mtDNA were $11.8 \%$ for the patient and $5.1 \%$ for the mother. In the renal biopsy specimen, the percentage was significantly higher, $65 \cdot 6 \%$. Although his sister's mtDNA appeared normal, Southern blotting using an internally positioned oligonucleotide as a probe showed that her mtDNA also contained a very small percentage of mutated mtDNA (data not shown).

Several lines of evidence suggest that the nephropathy was caused primarily by the mitochondrial mutation. First, although our patient developed diabetes mellitus, the development of proteinuria preceded that of glucosuria. Secondly, the results of laboratory tests were against incidental association of other types of nephritis/nephropathy. Thirdly, the estimated percentage of mutant mtDNA was higher in the renal biopsy specimen.

Recently Rötig et al ${ }^{1}$ reported a patient with a $2.6 \mathrm{~kb}$ deletion of mtDNA, who presented with tubulointerstitial nephritis. How a point mutation and a mtDNA deletion can give rise to a similar clinical presentation is unclear. However, any mutation in mtDNA is presumed to result in a decreased supply of ATP. Since renal tubules have ATP/dependent transport systems, it is not surprising that any dysfunction in the mitochondria causes tubulointerstitial dysfunction, which somehow leads to structural damage.

Another interesting aspect of this case was $\mathrm{GH}$ deficiency. Short stature is a common feature of patients with A $3243 \mathrm{G}$ mtDNA mutation $^{2}$ and our case suggests that GH deficiency could be the cause of short stature in these patients. Since the prognosis of mitochondrial diabetes is similar to that of other forms of diabetes, $\mathrm{GH}$ therapy could potentially improve the quality of life of these patients. Interestingly, a recent report by Bernardi $e t ~ a l^{3}$ showed that ATP sensitive $\mathrm{K}+$ channels are associated with the secretion of insulin by pancreatic islet cells and secretion of growth hormone by the anterior pituitary. Possibly, the decreased supply of ATP caused by the mtDNA mutation opened the channel, thereby decreasing the secretion of insulin and growth hormone.

We thank Dr Fujisawa at Fujisawa Clinic, Shiga, and Dr Hiraoka at Fukui Medical College, Fukui, Japan for instructions and helpful discussions.

1 Rötig A, Goutières F, Niaudet P, Rustin P, et al. Deletion of mitochondrial DNA in patient with chronic tubulointerstitial nephritis. $\mathcal{F}$ Pediatr 1995;126:597-601

2 Kishimoto $M$, Hashiramoto $M$, Araki $S$, et al. Diabetes mellitus carrying a mutation in the mitochondrial tRNA $^{\text {Leu(UUR) }}$ gene. Diabetologia 1995;38:193-200.

3 Bernardi H, De-Weille JR, Epelbaum J, et al. ATP-dependent $\mathrm{K}+$ channels sensitive to antidiabetic sulfonylureas are present in adenohypophysis and are involved in growth hormone release. Proc Natl Acad Sci USA 1993;90:1340-4. 